\title{
Pellet Fuelling of Tokamaks
}

\section{Andrew Cheetham, Abingdon}

(JET Joint Undertaking)

Over the past decade, the most successful method of fuelling tokamaks has been simply to inject the gas (deuterium or hydrogen) into the vacuum chamber via a fast acting, electronically controllable valve. This valve gives either a preprogrammed flow of gas, or is controlled by a servo-system that tracks the plasma density and maintains it at a pre-set level below the critical density at which the plasma current will disrupt, the density limit. To date this method of fuelling has worked adequately, but there are drawbacks that must be avoided as experiments approach fusion conditions.

A recent technique is to inject high speed pellets of frozen hydrogen isotopes directly into the plasma core. This method offers several advantages over that of gas puffing: the efficiency in fuel delivery is much higher; it can be used to control and peak the density profile; the pure fuel of the pellet dilutes the impurities in the plasma and the impurity influx from the material surfaces interacting with the plasma may be reduced.

The route by which an atom, introduced into the vacuum vessel by gas puffing, finds its way into the plasma involves several cycles of ionisation in the outer edges of the plasma, loss to the vessel wall and re-release by collision with another escaping particle. This process, known as recycling, is also responsible for the release of impurities from the wall into the plasma and an increase in the impurity content, measured by the effective charge of the plasma:

$$
Z_{\text {eff }}=\Sigma\left(n Z_{i}^{2}\right) / \Sigma\left(n Z_{i}\right)
$$

where $n_{i}$ and $Z_{i}$ are the density and the ionic charge of the $i$ th ion species. For a pure deuterium plasma $Z_{\text {eff }}=1$.

An important effect of the recycling process is that during gas injection, large quantities of the fuel (up to $80 \%$ ) end up embedded in the limiter and wall of the vacuum chamber, reducing the efficiency of gas fuelling to as low as 0.2. During the active phase of JET, where for safety and environmental reasons, minimization of the tritium inventory will be a prime consideration, this would be a serious handicap. Fuelling by pellet injection avoids the problem by depositing pure deuterium or tritium inboard of the recycling layer of the plasma. The fuelling efficiency of pellet injection is therefore much higher and if all the injected fuel is burnt in the reacting plasma, the tritium inventory question is much less severe.

On some tokamaks (e.g. ASDEX, DITE and $\mathrm{JT}-60$ ) the influx of impurities into the plasma is reduced through the use of a magnetic limiter or 'divertor' whereby the outermost magnetic flux surfaces are diverted into an exhaust chamber. Particles following these field lines are isolated from the bulk plasma, neutralised and pumped away. While such sys- tems remove some impurities before they reach the plasma they also prevent efficient fuelling by gas injection, and a much higher influx of fuel is required to attain the prescribed density. Again such problems are avoided by direct pellet fuelling of the plasma core.

A high plasma impurity content has several deleterious effects, most seriously high plasma radiation and a low density limit above which is the possibility of disruption. Fuelling by pellet injection deposits pure fuel directly inside the plasma not only fuelling but also reducing $Z_{\text {eff }}$ by diluting the impurities. With pellet injection the density limit for gas

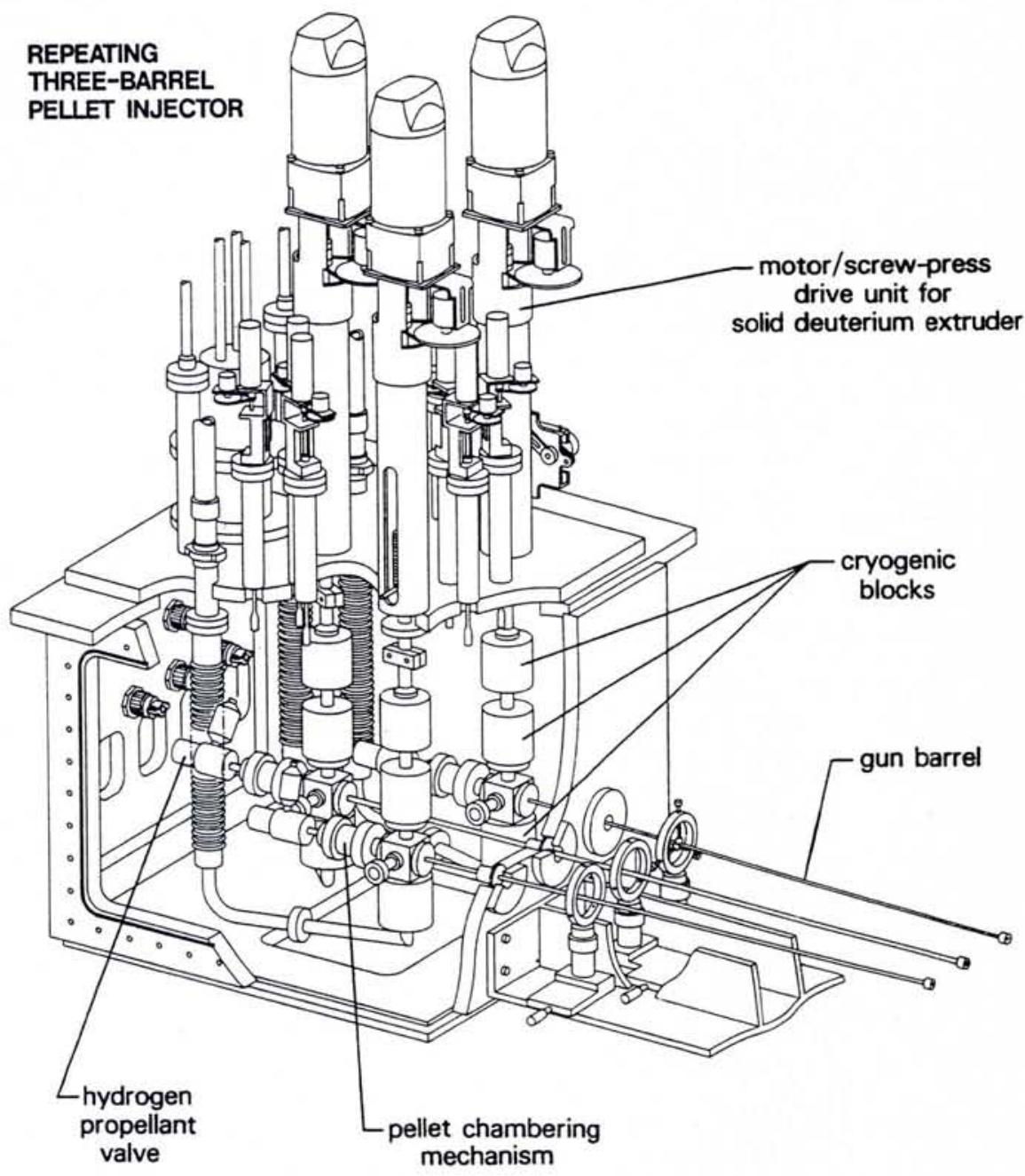

Fig. 1 - A cut-away diagram of the ORNL built multi-pellet launcher showing the major components of the three independent guns. 
fuelled discharges has been exceeded by up to $50 \%$.

A most important advantage of pellet fuelling is the possibility of controlling density profile by the judicious choice of pellet size and velocity. Under normal gas puffing conditions, the density profile is determined only by the local transport coefficients in the plasma, whereas it can be shown that the fusion yield will be enhanced if the profile is centrally peaked. Such a profile can be obtained by injecting pellets into the plasma core before applying auxiliary heating, such as ion cyclotron resonance heating (ICRH) or neutral beam injection (NBI).

Many experiments have successfully demonstrated the efficacy of fuelling by pellet injection although severe technological problems have to be surmounted if this success is to be repeated in fusion plasmas. Centrally fuelling a plasma at fusion temperatures will require pellet speeds greater than $10 \mathrm{~km} / \mathrm{s}$, far in excess of what is currently available. Concerted developmental work in pellet injector technology will, as a result, be needed.

\section{Current Technology}

The function of the pellet injector is to form a pellet of frozen fuel, accelerate the pellet without breaking it, and project it into the plasma. Developmental work over the past few years has led to two main types of injection system: the pneumatic type, where the pellet is blown out of a gun barrel with high pressure gas, and the centrifugal type where the pellet is effectively thrown from a spinning rotor.

The pneumatic injector has been separately developed at Ris $\emptyset$ National Laboratory, Oak Ridge National Laboratory (ORNL), Max-Planck Institut für Plasmaphysik (IPP) Garching and CENG Grenoble. All of these guns, except the Grenoble version where the fuel is condensed directly in the barrel, work on the principle of extruding solid deuterium, forming the pellet by either cutting or moulding, and then blowing it out with helium or hydrogen at pressures up to 10 $\mathrm{MPa}$. The pellets are usually cylindrical, of sizes up to diameter $=$ length $=6 \mathrm{~mm}$ and have been fired with velocities up to $1.6 \mathrm{~km} / \mathrm{s}$. A major concern with this type of injector is to prevent the propellant gas from following the pellet into the plasma. This can be achieved by installing a large multi-volume, differentially pumped vessel between the end of the barrel and the plasma vacuum chamber. In the case of single shot injectors, a fast closing valve can also be used.

The advantage of the centrifugal injector is that it can achieve a very high

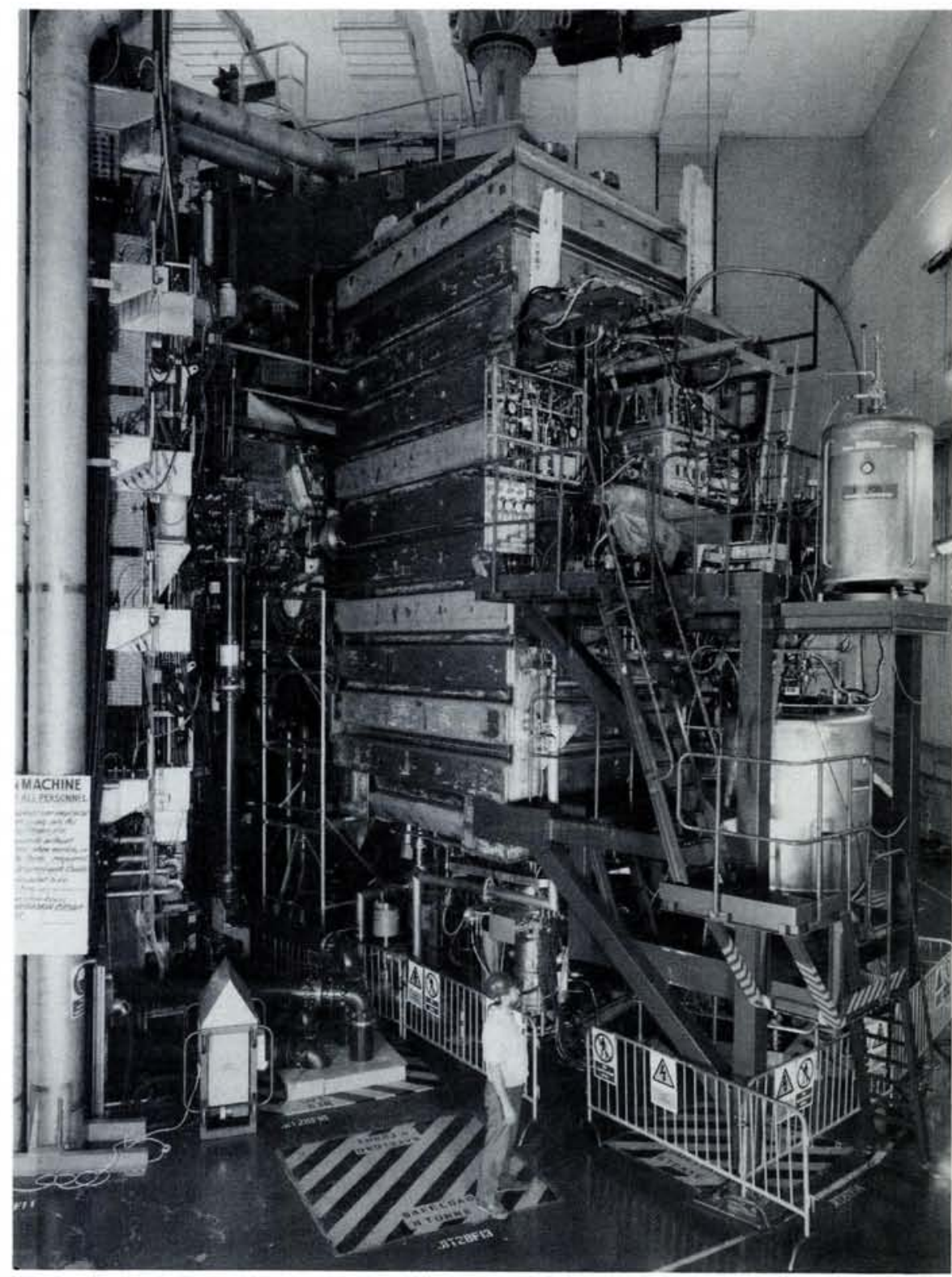

Fig. 2 - The JET pellet injection system as installed. The large steel box is the propellant gas trapping volume, the launcher is installed on the back of the box, just above the top platform.

repetition rate (up to $50 \mathrm{~Hz}$ has been obtained at IPP), but this is compromised by the rather low pellet velocities, $<$ $800 \mathrm{~m} / \mathrm{s}$. This type of injector comprises a spinning rotor with a raised bar running in a semi-circle from the centre to the edge. The fuel is extruded perpendicular to the rotor and close to the centre where the raised bar cuts off a pellet that then accelerates round the semi-circle to be thrown off the edge at twice the edge velocity. In theory, much higher pellet velocities could be obtained, but the maximum velocity is limited by the stress on the rotor and by the strength of the deuterium and hence its ability to survive the rigours of the cutting and throwing process. Although high repetition rates are possible, control is difficult as fuelling rate and pellet speed depends on the rotor speed which cannot be changed quickly.
There are other acceleration systems being developed that are designed to accelerate the pellet in a controlled manner in order to attain a higher muzzle velocity without the pellet breaking up. These include a two stage pneumatic gun with adiabatically heated propellant gas (Ernst-Mach-Institut (EMI), Freiburg, FRG), an arc driven gun with a tailored discharge to apply the pressure as a preprogrammed, controlled pulse, (Ris $\emptyset$ ); both of these projects are supported by JET. Furthermore, there is work on a two stage gun (Frascati/Milano), an arc driven and an electron beam driven gun at ORNL, and a rail gun at the University of Illinois, USA. The ultimate aim of all these techniques is to be able to fire a pellet at velocities $\gg 2 \mathrm{~km} / \mathrm{s}$. The maximum velocity so far obtained from the two stage gun (EMI), is $\cong 2.6 \mathrm{~km} / \mathrm{s}$ with unsupported deuterium and $\cong 3.8 \mathrm{~km} / \mathrm{s}$ 
with deuterium supported in a plastic boot which must be stripped off before the pellet enters the plasma.

\section{JET Multi-Pellet Injector}

The pellet injector currently operating on JET comprises the pellet launcher and the machine interface, the largest part of which is the differential pumping system. The apparatus was built by JET and ORNL who installed the launcher under a joint agreement between JET and the US Department of Energy. Fig. 1 is a cut-away diagram showing the main components. It is a three barrel repeating, pneumatic system comprising three separate and independent guns capable of firing $2.7 \mathrm{~mm}, 4.0 \mathrm{~mm}$ and $6.0 \mathrm{~mm}$ pellets with repetition rates of up to $5 \mathrm{~Hz}, 2.5 \mathrm{~Hz}$ and $1 \mathrm{~Hz}$ respectively. Each of the guns operates on the same principle: a reservoir of solid deuterium is formed and when required, is extruded as a ribbon down past the barrel end. The pellet is formed immediately before firing by an electromechanical punch which punches the pellet from the moving ribbon into the end of the barrel from where it is blown out with hydrogen at a pressure of up to $10 \mathrm{MPa}$. The maximum repetition rate is a function of the size of the pellet and the ribbon speed which in turn is limited by the extrusion speed. Each gun can be operated independently at the stated repetition rate for a $20 \mathrm{~s}$ period. The average pellet velocity under these conditions is $\cong 1.3 \mathrm{~km} / \mathrm{s}$, although $1.5 \mathrm{~km} / \mathrm{s}$ can be attained in single shot mode.

With the possibility of firing so many pellets in a short time, the method of trapping the considerable volume of propellant gas before it reaches the plasma chamber has had to be carefully considered. The method chosen is to fire the pellets through a $50 \mathrm{~m}^{3}$ expansion chamber equipped with a $8 \times 10^{6} \mathrm{I} / \mathrm{s}$, liquid helium cooled, cryo-condensation pump. The pellets exit this volume along narrow flow restrictor tubes $16 \mathrm{~cm}$ diameter, $1 \mathrm{~m}$ long) to further decouple the buffer chamber from the JET vacuum vessel. A photograph of the injector assembly is shown in Fig. 2. The actual pellet launcher (Fig. 1) is barely visible on the rear of the buffer volume above the upper platform. The expansion volume and pump combination is over-dimensioned for the present injector; it has been designed to cope with the predicted gas load from the high speed injectors under development.

The entire operation of the injector is by remote control from the JET control room via a CAMAC-based user interface to a programmable logic controller. Once the pellet firing sequence has been pro- grammed in, the operation during a pulse is fully automatic. A real-time protection system monitors the important plasma parameters and will inhibit pellet injection if it would endanger operation, by producing disruption for example. A servo-system to choose the pellet size and firing sequence in real time to obtain a pre-programmed density level is under development.

The following monitors and diagnostics have been fitted to the pellet injector: remote TV monitoring of the extruders to check visually the quality of the solid fuel; fibre optic pellet detection for timing; and high speed flash photography of the moving pellet as it leaves the barrel to identify its quality. Inside the buffer volume, the pellet passes through two tuned micro-wave cavities, separated by ca $0.5 \mathrm{~m}$, perturbing the resonance. It is observed as a pulse whose amplitude is a function of pellet mass. The temporal separation of the two signals is used to calculate the pellet velocity.

\section{Pellet Injection Experiments}

The results of pellet injection experiments can be considered on three time scales:

(a) The penetration and ablation of the pellet in the plasma, $\leq 1 \mathrm{~ms}$.

(b) The time it takes for the plasma to recover from the perturbation due to the pellet, $\leq 400 \mathrm{~ms}$.

(c) Longer term effects on the plasma, $\geq 400 \mathrm{~ms}$.

In this section measurements of various plasma parameters made on these three time scales will be described.

(a) Pellet ablation on JET is monitored by measuring the $\mathrm{H}_{\alpha}$ and soft $X$-ray radiation from the plasma during pellet penetration. From these data the precise penetration depth of the pellet can be determined and compared with values calculated from various models. A simple electron bombardment model gives

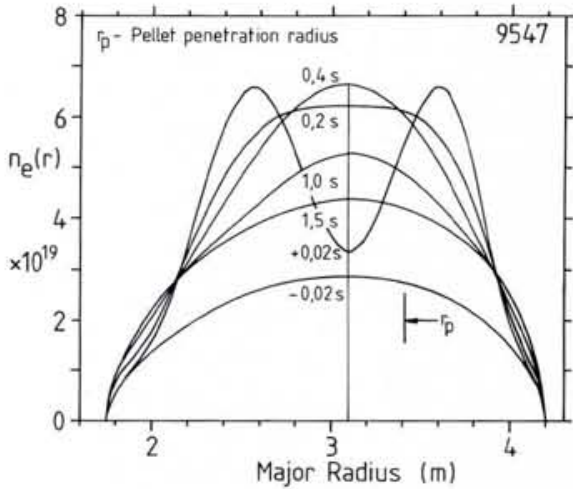

Fig. 3 - The radial profile of the electron density after pellet injection showing the evolution from a hollow profile immediately after ablation, to a more peaked profile around half a second later. The times indicated are relative to the injection time.

an ablation rate far higher than that which is observed in practice, whereas good agreement with experiment can be obtained using a model developed at ORNL based on the assumption that the pellet becomes enclosed in a shell of neutral gas outside which is a shell of ionised gas producing a very high temperature gradient. Pellet speed is relatively slow in relation to particle speeds and so can be considered stationary in the flux tube that contains it for the purpose of calculating the energy deposition. Subsquent iterations as the pellet decreases in size and penetrates deeper into the plasma allows the pellet ablation to be simulated, the crucial parameters being the depths ascribed to the neutral gas and plasma shielding layers. The penetration depends on velocity, the plasma density profile and, most critically, the plasma electron temperature profile.

(b) The immediate effect of the pellet on the plasma electron density is shown in Fig. 3 where the radial profile of the density, deduced from FIR laser interferometry, is plotted at several times before and after the injection of a pellet.

4th April - 14th April 1989

Geilo, Norway

\section{NATO Advanced Study Institute} on

\section{Phase Transitions in Soft Condensed Matter}

Topics of Lectures

$\begin{array}{lllll}\text { Aggregation } & \text { Gelation } & \text { Wetting } & \text { Liquid crystals } & \text { Microemulsions } \\ \text { Condensation } & \text { Interfaces } & \text { Colloids } & \text { Membranes } & \text { Polymers } \\ \text { Fluctuations } & \text { Melting } & \text { Gels } & \text { Micelles } & \text { Surfactants }\end{array}$

Topics will cover both theory and experiments. Open to participants from all countries. Deadline for application is 15 January 1989.

Information: Gerd Jarrett, Dept. of Physics, Institute for Energy Technology, POB 40, N-2007 Kjeller, Norway. Tel.: ++ 47 (6) 812560 
In this case the pellet penetrates just past mid-radius resulting immediately in a hollow density profile. The profile fills in over $200 \mathrm{~ms}$ and recovers to a peaked profile in $\cong 400 \mathrm{~ms}$ before the density begins to decay.

The temporal evolution of other plasma parameters after pellet injection is shown in Fig. 4. Concomitant with the rise in the density is a sharp fall in the electron and ion temperatures. Recovery begins immediately with the density slowly falling and the temperature rising but on a faster time scale such that there is a net increase in plasma energy that peaks some $700 \mathrm{~ms}$ after injection. The effect on the impurity content is that $Z_{\text {eff }}$ is reduced from 2.5 down to 1.6 , agreeing well with the value calculated from simple dilution of the existing plasma by the injection of pure deuterium. The neutron yield (D-D reaction rate) also increases in close agreement with the rise in deuterium density and temperature.

(c) On a longer time scale the net effect of the pellet is to produce a clean, high density plasma that lasts for $\cong 0.5$ - $1 \mathrm{~s}$ after injection before most parameters return approximately to their prepellet values. Under certain plasma conditions the effect of the pellet is longer lived. The application of high power ICRF heating to such a centrally peaked

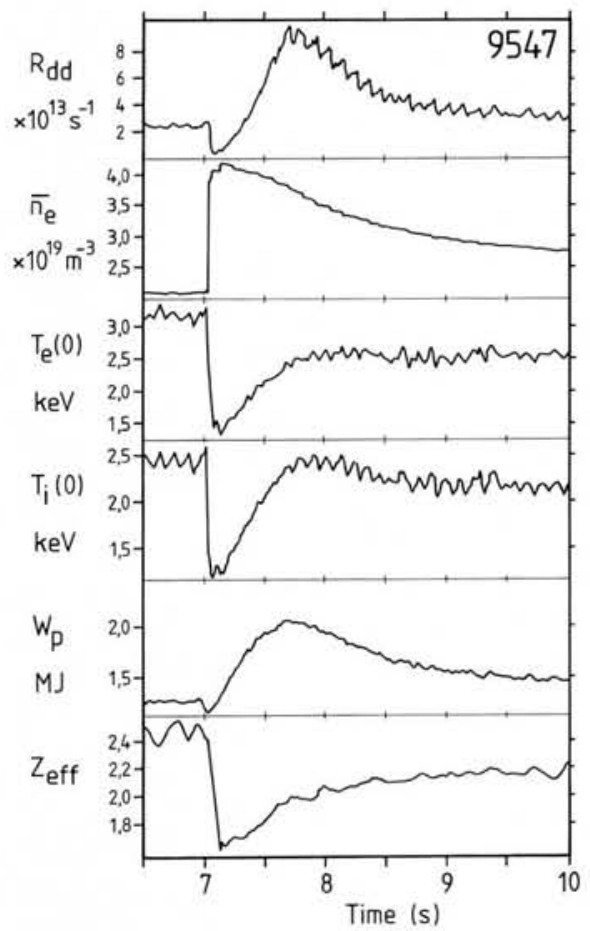

Fig. 4 - Temporal evolution of several plasma parameters after pellet injection. The $D-D$ reaction rate $R_{d d}$, average electron density $\bar{n}_{e}$, central electron and ion temperatures $T_{e}$ and $T_{i}$, total plasma energy content $W_{p}$ and the impurity content $Z_{\text {eff }}$. This is a 3 MA discharge with no auxiliary heating, the pellet was injected at $7 \mathrm{~s}$.

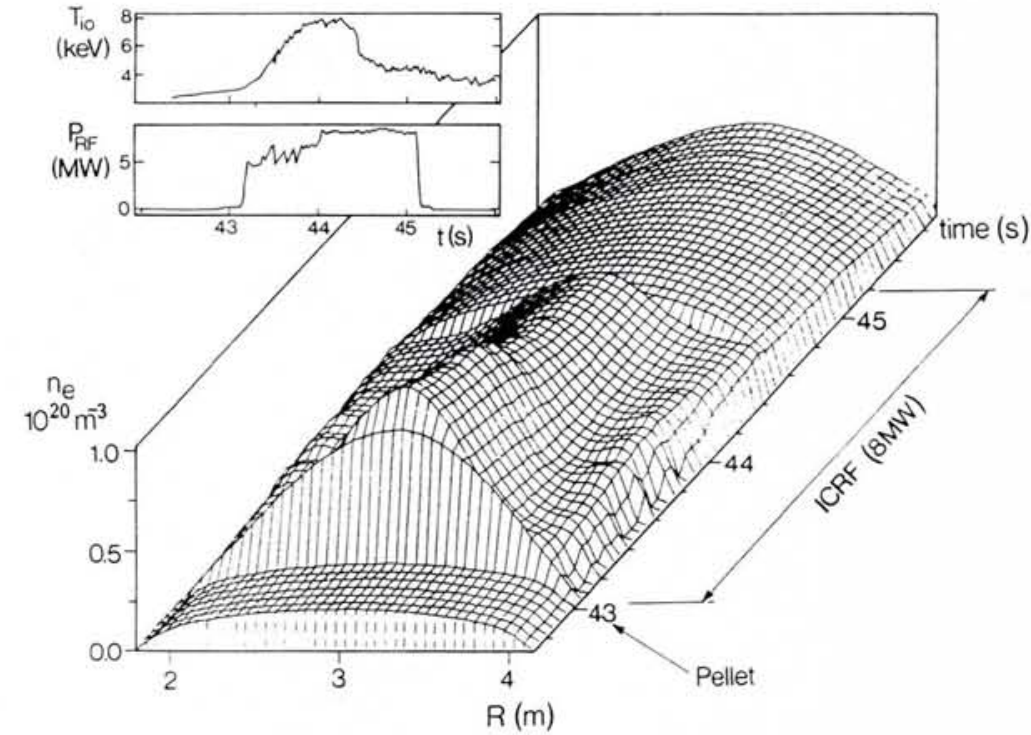

Fig. 5 - Temporal and spatial evolution of the electron density after pellet injection to the centre of the plasma followed immediately by high power ICRF heating. Also shown are the temporal evolution of the ion temperature and the ICRF input power.

density profile (Fig. 5), obtained when the pellet penetrates to the centre of the plasma, results in extremely high electron and ion temperatures and, coupled with the high deuteron concentration produces a greatly enhanced fusion neutron yield.

\section{Conclusions}

The results of recent experiments on JET have demonstrated the efficacy of pellet fuelling of high temperature plasmas. The direct fuelling of the core produces a pure, dense plasma with a centrally peaked density profile. The application of auxiliary heating to such plasmas can produce a long lived ( $>1 \mathrm{~s}$ ) dense, hot core giving a high fusion yield. Further work will concentrate on maintaining and extending the life time of these plasma conditions. Developmental work will continue on injector technology to increase the pellet speed for effective penetration of fusion temperature plasmas.

\section{Acknowledgment}

The author would like to acknowledge A. Gondhalekar and P. Kupschus for their collaboration and helpful comments, the pellet injection and ICRH groups at JET and G. Schmidt and the US pellet injection team, for supplying the data.

\section{FURTHER READING}

Details of the JET Pellet Injector Milora S et al., Proceedings of the IEEE Symposium on Fusion Engineering, Monterey, October $1987 \mathrm{Vol}$. 2, page 784.

Kupschus P. et al., ibid Vol. 2, page 780.

Advances in High Speed Pellet Development Sonnenberg K. et al., ibid Vol. 2, page 1208.

\section{Experimental Results}

Gondhalekar A. et al., Proc. of the 11th IAEA Conf. on Plasma Physics and Controlled Nuclear Fusion Research, Kyoto 1986 Vol. 3, page 457.

Kupschus P. et al., Proc. 15th European Conf. on Controlled Fusion and Plasma Heating, Dubrovnik, May 1988 Vol. 12b, part 1, page 143.

Milora S. et al., ibid, page 147.

\section{Physical Society of Japan}

The 44th Annual Meeting of the Physical Society of Japan is to be held at Tokai University, Hiratshuka-shi, Kanagawa-ken from 28-31 March 1989. Application deadline for presentation of papers is $14 \mathrm{Dec}$. 1988. As the Society is a Collaborating Society of EPS, IOMs enjoy the same rights as members of the PSJ. Enquiries should be addressed to:

The Physical Society of Japan Room 211, Kikai-Shinko Building 3-5-8 Shiba-Koen, Minato-Ku Tokyo 105, Japan Tel.: (3) 4342671
Through the collaboration agreement concluded between EPS and the PSJ in 1987, Individual Ordinary Members of EPS may also subscribe to the Journal of the Physical Society of Japan at the same rate as members of the PSJ.

For Volume 58 (1989) the annual rate for Europe is: $Y 14000$ surface mail

$$
Y 39000 \text { airmail }
$$

Questions regarding subscriptions should be sent to the Physical Society of Japan at the address given in the adjacent column. 